\title{
Health-related quality of life in patients with Gilles de la Tourette syndrome at the transition between adolescence and adulthood
}

\author{
Paola R. Silvestri ${ }^{1} \cdot$ Flavia Chiarotti $^{2}$ - Valentina Baglioni ${ }^{1} \cdot$ Valeria Neri $^{1}$. \\ Francesco Cardona $^{1} \cdot$ Andrea E. Cavanna $^{3,4,5}$
}

Received: 19 June 2016/ Accepted: 20 July 2016/Published online: 25 July 2016

(c) The Author(s) 2016. This article is published with open access at Springerlink.com

\begin{abstract}
Gilles de la Tourette syndrome (GTS) is a neurodevelopmental condition characterised by tics and comorbid behavioural problems, affecting predominantly male patients. Tic severity typically fluctuates over time, with a consistent pattern showing improvement after adolescence in a considerable proportion of patients. Both tics and behavioural co-morbidities have been shown to have the potential to affect patients' health-related quality of life (HR-QoL) in children and adults with persisting symptoms. In this study, we present the results of the first investigation of HR-QoL in patients with Gilles de la Tourette syndrome at the transition between adolescence and adulthood using a disease-specific HR-QoL measure, the Gilles de la Tourette Syndrome-Quality of Life-Children and Adolescents scale. Our results showed that patients with GTS and
\end{abstract}

Andrea E. Cavanna

a.cavanna@ion.ucl.ac.uk

1 Department of Pediatrics and Child Neuropsychiatry, Sapienza University, Rome, Italy

2 Department of Cell Biology and Neuroscience, National Institute of Health, Rome, Italy

3 Department of Neuropsychiatry, The Barberry National Centre for Mental Health, BSMHFT and University of Birmingham, 25 Vincent Drive, Birmingham B15 2FG, UK

4 Sobell Department of Motor Neuroscience and Movement Disorders, Institute of Neurology and University College London, London, UK

5 School of Life and Health Sciences, Aston Brain Centre, Aston University, Birmingham, UK more severe co-morbid anxiety symptoms reported lower HR-QoL across all domains, highlighting the impact of anxiety on patient's well-being at a critical stage of development. Routine screening for anxiety symptoms is recommended in all patients with GTS seen at transition clinics from paediatric to adult care, to implement effective behavioural and pharmacological interventions as appropriate.

Keywords Anxiety - Gilles de la Tourette syndrome . Health-related quality of life - Tics

\section{Introduction}

Gilles de la Tourette syndrome (GTS) is a neurodevelopmental disorder characterised by multiple motor and phonic tics [1]. The average age at onset of GTS is around 7 years, and a significant reduction of tic severity after adolescence occurs in about one-third of cases [1]. Comorbid behavioural problems are reported by the majority of patients with GTS, especially attention-deficit and hyperactivity disorder (ADHD), obsessive-compulsive disorder (OCD), anxiety and affective disorders [1]. Clinical studies in children and adults with GTS have shown that both tics and behavioural symptoms can affect patients' health-related quality of life (HR-QoL) [2]. To date, few data are available about the impact of GTS on HR-QoL at the transition between adolescence and adulthood, a crucial age for patients with tic disorders. We, therefore, set out to investigate HR-QoL and its determinants in a clinical sample of adolescents and young adults with GTS using a disease-specific HR-QoL measure, alongside a comprehensive battery of psychometric instruments [3]. 


\section{Methods}

\section{Participants}

Twenty-two consecutive patients (5 girls) with a diagnosis of GTS and a mean age of 18 years [age range 15-19 years; standard deviation (SD) 1 year] were recruited from the Child and Adolescent Neuropsychiatry Outpatient Unit of the Sapienza University in Rome, Italy. Each participant/parent provided written informed consent before enrolment. Eight out of the 22 patients had no comorbid psychiatric disorders, 8 were diagnosed with OCD, 4 with ADHD, and 2 with both OCD and ADHD. The mean age at tic onset was 8 years (SD 3 years). Eighteen patients were on pharmacotherapy ( 8 with atypical antipsychotics, 1 with typical antipsychotics, 2 with antidepressants, 7 with other medications).

\section{Procedure}

Specialist clinicians with extensive experience in tic disorders collected demographic and clinical information and assessed the severity of tics using the Yale Global Tic Severity Scale (YGTSS) [4], a clinician-rated instrument that assesses the number, frequency, intensity, complexity and interference of motor and phonic tics. YGTSS total tic severity scores range from 0 (no tics) to 50 (extremely severe tics). Each participant was subsequently asked to complete the following psychometric instruments to assess HR-QoL, premonitory urges, and co-morbid behavioural symptoms:

- Gilles de la Tourette Syndrome-Quality Of Life-Children and Adolescents scale (C\&A-GTS-QOL) [5]

The C\&A-GTS-QOL is a 27 -item, self-report questionnaire that assesses HR-QoL in young patients with tic disorders, encompassing 4 areas of HR-QoL: psychological, physical/activities of daily living, obsessive-compulsive, and cognitive domains. Each item is rated on a 5-point Likert-type scale and higher scores indicate worse HR-QoL. The instrument includes a Visual Analogue Scale used to express the extent of self-satisfaction about life (higher scores indicate higher satisfaction).

- Premonitory Urge for Tics Scale (PUTS) [6]

The PUTS is a 10-item self-report scale that assesses premonitory urges to tic. Items from 1 to 9 receive a score ranging from 1 (never) to 4 (always). Higher scores indicate the presence of more severe urges.

- State-Trait Anxiety Inventory-form Y (STAI-Y) [7]

The STAI is a 40-item, self-report questionnaire assessing state (STAI-Y1) and trait anxiety (STAIY2) in subjects older than 12 years. Items are rated on a 4-point Likert-type scale, and higher scores indicate more severe symptoms.

- Beck Depression Inventory-II (BDI-II) [8]

The BDI-II is a 21-item, self-report questionnaire assessing depression in subjects older than 13 years. Items are rated on a 4-point Likert-type scale, and higher scores indicate more severe symptoms.

\section{Statistical analysis}

Participants were divided into subgroups based on the following grouping variables: (a) gender (male vs female); (b) tic severity (YGTSS score $<10$ vs $\geq 10$ ); (c) premonitory urge severity (PUTS score $<25$ vs $\geq 25$ ); (d) anxiety state (STAI-Y $1<75$ vs $\geq 75$ th centile) and trait (STAI$\mathrm{Y} 2<75$ vs $\geq 75$ th centile); (e) affective symptoms (BDIII $<90$ vs $\geq 90$ th centile). Differences in GTS-QOL-C\&A scores among subgroups were assessed using the MannWhitney $U$ test. Statistical analyses were performed using the STATA Statistical Software, Version 8.1.

\section{Results}

In our sample, C\&A-GTS-QOL total and subscale scores were lower in patients who reported more severe state and trait anxiety (Table 1).

Analysis of C\&A-GTS-QOL VAS scores confirmed these findings, as patients with lower levels of anxiety consistently reported higher overall satisfaction with life. The negative effects of anxiety symptoms on HR-QoL were particularly significant in the psychological domain, whereas the obsessional domain of HR-QoL was also affected by the presence of more severe urges to tic and affective symptoms. Patients with at least one co-morbid psychiatric diagnosis reported higher C\&A-GTS-QOL scores compared to patients with GTS only, although these differences did not reach statistical significance $(p=0.09)$. No significant differences in C\&A-GTS-QOL scores were observed between male and female patients, or between patients with lower and higher tic severity.

\section{Discussion}

We conducted the first study focusing on HR-QoL and its clinical correlates in patients with GTS at the transition age between adolescence and adulthood. This is a particularly delicate age for patients with tic disorders, as the persistence of symptoms into early adulthood suggests that the tic disorder might persist throughout life as a potentially stigmatising condition. Interestingly, in our 
Table 1 Health-related quality of life ratings (mean values \pm SD) in patients with Gilles de la Tourette syndrome grouped by clinical variables

\begin{tabular}{|c|c|c|c|c|c|c|}
\hline & $\begin{array}{l}\text { C\&A-GTS- } \\
\text { QOL total }\end{array}$ & $\begin{array}{l}\text { C\&A-GTS-QOL } \\
\text { psychological }\end{array}$ & $\begin{array}{l}\text { C\&A-GTS-QOL } \\
\text { obsessional }\end{array}$ & $\begin{array}{l}\text { C\&A-GTS-QOL } \\
\text { cognitive }\end{array}$ & $\begin{array}{l}\text { C\&A-GTS-QOL } \\
\text { physical/ADL }\end{array}$ & $\begin{array}{l}\text { C\&A-GTS- } \\
\text { QOL VAS }\end{array}$ \\
\hline BDI-II $(p)$ & 0.0767 & 0.1111 & 0.0452 & 0.4590 & 0.1718 & 0.0865 \\
\hline $\operatorname{Neg}(n=16)$ & $26.56 \pm 17.64$ & $29.63 \pm 17.10$ & $19.06 \pm 20.10$ & $31.44 \pm 26.16$ & $24.00 \pm 20.45$ & $74.06 \pm 17.34$ \\
\hline Pos $(n=6)$ & $43.00 \pm 17.47$ & $47.50 \pm 20.00$ & $44.17 \pm 29.40$ & $35.67 \pm 18.82$ & $39.83 \pm 24.64$ & $54.50 \pm 22.12$ \\
\hline PUTS $(p)$ & 0.0559 & 0.4104 & 0.0305 & 0.0804 & 0.1714 & 0.4688 \\
\hline $\operatorname{Neg}(n=14)$ & $24.93 \pm 12.19$ & $31.21 \pm 14.86$ & $15.00 \pm 13.01$ & $25.86 \pm 19.73$ & $21.79 \pm 15.03$ & $70.14 \pm 23.39$ \\
\hline Pos $(n=8)$ & $41.75 \pm 23.91$ & $40.25 \pm 25.34$ & $45.00 \pm 30.12$ & $44.38 \pm 27.55$ & $39.75 \pm 28.84$ & $66.25 \pm 14.33$ \\
\hline STAI-Y1 $(p)$ & 0.0064 & 0.0010 & 0.0106 & 0.0662 & 0.1349 & 0.0143 \\
\hline $\operatorname{Neg}(n=14)$ & $22.00 \pm 13.34$ & $24.79 \pm 14.00$ & $15.00 \pm 18.29$ & $23.14 \pm 15.32$ & $22.36 \pm 19.41$ & $77.50 \pm 15.66$ \\
\hline Pos $(n=7)$ & $46.43 \pm 17.79$ & $52.71 \pm 15.91$ & $44.29 \pm 26.37$ & $43.57 \pm 25.51$ & $38.71 \pm 26.26$ & $53.86 \pm 20.27$ \\
\hline STAI-Y2 $(p)$ & 0.0228 & 0.0510 & 0.0096 & 0.1543 & 0.1001 & 0.0104 \\
\hline $\operatorname{Neg}(n=14)$ & $23.14 \pm 13.36$ & $28.00 \pm 15.68$ & $14.64 \pm 15.50$ & $24.50 \pm 17.00$ & $21.07 \pm 16.99$ & $77.14 \pm 16.49$ \\
\hline $\operatorname{Pos}(n=7)$ & $44.14 \pm 20.90$ & $46.29 \pm 22.17$ & $45.00 \pm 29.15$ & $40.86 \pm 25.48$ & $41.29 \pm 27.77$ & $54.57 \pm 19.78$ \\
\hline
\end{tabular}

Statistically significant values ( $p \leq 0.05$; Mann-Whitney $U$ test) shown in bold

$C \& A-G T S-Q O L$ Gilles de la Tourette Syndrome-Quality Of Life-Children and Adolescents scale, $A D L$ activities of daily living, VAS Visual Analogue Scale, BDI-II Beck Depression Inventory-II; PUTS Premonitory Urge for Tics Scale, STAI-Y1 State-Trait Anxiety Inventory-State Anxiety, STAI-Y2 State-Trait Anxiety Inventory-Trait Anxiety

clinical sample, neither gender nor tic severity was associated with decreased HR-QoL, and the impact of premonitory urges and affective symptoms was limited to the obsessional domain of HR-QoL. However, we found a significantly lower report of $\mathrm{HR}-\mathrm{QoL}$ in patients with higher levels of state and trait anxiety. These results are consistent with findings from previous studies using generic HR-QoL instruments in adult patients with GTS, which highlighted a pervasive effect of anxiety on HRQoL [9] and showed that anxiety symptoms, as well as affective symptoms, moderate the relationship between tic severity and functional impairment, such that stronger relationships are documented in participants with elevated anxiety or depression [10]. The effects of psychiatric comorbidities, especially ADHD and OCD, on the HR-QoL of patients within this age group deserve further investigation, based on our preliminary findings [11]. Limitations of the present study include the relatively small sample size (especially with regard to the subgroup of patients with GTS only), referral bias, use of self-report measures and retrospective design. Our findings highlight the crucial role that anxiety symptoms play in influencing the psychological well-being of patients with GTS from adolescence to early adulthood. Routine screening for anxiety symptoms is recommended in all patients with GTS seen at transition clinics from paediatric to adult care, to implement effective behavioural and pharmacological interventions as appropriate.

Acknowledgments The authors express their gratitude to the Tourette Association of America and Tourettes Action-UK for their continuing support.

\section{Compliance with ethical standards}

Ethical approval The study was approved by the local Ethics Committee and written informed consent was obtained from all subjects prior to enrolment. All procedures performed in studies involving human participants were in accordance with the ethical standards of the institutional and/or national research committee and with the 1964 Helsinki declaration and its later amendments or comparable ethical standards.

Conflict of interest The authors declare no potential conflicts of interest with respect to the research, authorship and/or publication of this article.

Funding The authors received no financial support for the research, authorship and/or publication of this article.

Open Access This article is distributed under the terms of the Creative Commons Attribution 4.0 International License (http://crea tivecommons.org/licenses/by/4.0/), which permits unrestricted use, distribution, and reproduction in any medium, provided you give appropriate credit to the original author(s) and the source, provide a link to the Creative Commons license, and indicate if changes were made.

\section{References}

1. Cavanna AE, Seri S (2013) Tourette's syndrome. Br Med J 347:f4964

2. Cavanna AE, David K, Bandera V, Termine C, Balottin U, Schrag A, Selai C (2013) Health-related quality of life in Gilles de la Tourette syndrome: a decade of research. Behav Neurol 27:83-93

3. Cavanna AE, Luoni C, Selvini C, Blangiardo R, Eddy CM, Silvestri PR, Calì PV, Gagliardi E, Balottin U, Cardona F, Rizzo R, Termine C (2013) Disease-specific quality of life in young patients with Tourette syndrome. Pediatr Neurol 48:111-114 
4. Leckman JF, Riddle MA, Hardin MT, Ort SI, Swartz KL, Stevenson J, Cohen DJ (1989) The Yale Global Tic Severity Scale: initial testing of a clinician-rated scale of tic severity. J Am Acad Child Adolesc Psychiatry 28:566-573

5. Cavanna AE, Luoni C, Selvini C, Blangiardo R, Eddy CM, Silvestri PR, Calì PV, Seri S, Balottin U, Cardona F, Rizzo R, Termine C (2013) The Gilles de la Tourette Syndrome-Quality of Life Scale for children and adolescents (C\&A-GTS-QOL): development and validation of the Italian version. Behav Neurol 27:95-103

6. Woods DW, Piacentini J, Himle MB, Chang S (2005) Premonitory Urge for Tics Scale (PUTS): initial psychometric results and examination of the premonitory urge phenomenon in youths with tic disorders. J Dev Behav Pediatr 26:397-403

7. Spielberger CD, Gorsuch RL, Lushene R, Vagg PR, Jacobs GA (1983) Manual for the state-trait anxiety inventory. Consulting Psychologists Press, Palo Alto
8. Beck AT, Steer RA, Brown GK (1996) Beck depression inventory-second edition manual. The Psychological Corporation, San Antonio

9. Elstner K, Selai CE, Trimble MR, Robertson MM (2001) Quality of Life (QOL) of patients with Gilles de la Tourette's syndrome. Acta Psychiatr Scand 103:52-59

10. Lewin AB, Storch EA, Conelea CA, Woods DW, Zinner SH, Budman CL (2011) The roles of anxiety and depression in connecting tic severity and functional impairment. J Anx Disord 25:164-168

11. Eddy CM, Cavanna AE, Gulisano M, Calì P, Robertson MM, Rizzo R (2012) The effects of comorbid obsessive-compulsive disorders and attention-deficit hyperactivity disorder on quality of life in Tourette syndrome. J Neuropsychiatry Clin Neurosci $24: 458-462$ 